\title{
Developmental changes in shortening of pro-saccade reaction time while maintaining neck flexion position
}

\author{
Kenji Kunita ${ }^{1 *}$, Katsuo Fujiwara ${ }^{2}$, Naoe Kiyota ${ }^{3}$, Chie Yaguchi $^{3}$ and Takeo Kiyota ${ }^{1}$
}

\begin{abstract}
Background: We investigated developmental changes in shortening of pro-saccade reaction time while maintaining neck flexion.

Methods: Subjects comprised 135 children (3-14 years) and 29 young adults (19-23 years). Children were divided into six groups in 2-year age strata. Pro-saccade reaction tasks for $30 \mathrm{~s}$ were performed in neck rest and flexion positions. Reaction times under each position were averaged in every 10-s period.

Results: Under neck rest position, reaction time in the 0-10 s period was significantly longer in the 3-to 4-year-old group than in the 5- to 6-year-old group and above. No significant age effect was found for reaction time in the 0-10 s period in the 5- to 6-year-old group and above. Although a significant effect of neck flexion was not observed until the 9- to 10-year-old group, significant shortening of reaction time with neck flexion was found in the 11- to 12-year-old group and above. Furthermore, this shortening was maintained until the first 20-s period in the 11- to 12-year-old group and during the entire $30 \mathrm{~s}$ in the 13- to 14-year-old and above.

Conclusions: These results suggest that brain activation with the maintenance of neck flexion, related to shortening of the pro-saccade reaction time, was found from a later age of approximately 11 years and above, compared with the age at which information-processing function in the pro-saccade was enhanced. In addition, brain activation with the maintenance of neck flexion was sustained longer with age.
\end{abstract}

Keywords: Development, Pro-saccade reaction time, Brain activation, Neck flexion

\section{Background}

In daily life, saccadic eye movements are often used to quickly and precisely shift the gaze to an appearing visual target. Such eye movement is called visually guided saccade, or pro-saccade. The pro-saccade is controlled only via the supraspinal pathways, including the lateral genicular body, occipital cortex, posterior parietal cortex, parietal eye field, frontal eye field, superior colliculus, and reticular formation [1-5]. Information-processing time in the neural pathway for the pro-saccade has been investigated using the reaction time method. Pro-saccade reaction time changes with development, shortening from early childhood to

\footnotetext{
*Correspondence: k-kunita@ts.siu.ac.jp

'Department of Sports Instruction, Faculty of Sports and Human, Sapporo International University, 4-1-4-1 Kiyota, Kiyota-ku, Sapporo 004-8602, Japan Full list of author information is available at the end of the article
}

approximately 6-8 years old, then reaching a similar level to that in young adults [6-13]. One of the neurological factors causing these age-related changes in childhood is probably myelination in the neural pathway of pro-saccade $[8,14]$.

The saccade system is also reportedly supported and/ or promoted by the brain activation system [15-18]. We have previously reported that maintaining a flexed neck position, which constitutes part of the dynamic posture [19], leads to shortening of the pro-saccade reaction time [20, 21]. This shortening was observed without the chin resting on a stand, so the neck extensors would have been activated [20]. We also reported that pro-saccade reaction time shortened with voluntary isometric contraction of the superficial neck extensors by shoulder girdle elevation [22], or with vibration stimulation to the trapezius, one of the superficial neck 
extensors [23, 24]. These findings suggest that the shortening with maintaining neck flexion posture is attributable to an ascending brain activation system in tandem with increases in muscle afferent information [25-27]. The following changes in other physiological indices also support brain activation during neck flexion: 1) shortening of limb reaction times and latencies of visual, auditory and somatosensory evoked potentials [28, 29]; 2) increased amplitudes of auditory evoked potentials and event-related potentials associated with motor preparation and cognition [28-30]; and 3) shortened latencies and increased amplitudes of motor evoked potentials evoked by transcranial magnetic stimulation [31].

We have demonstrated that such shortening of the reaction time was affected by training $[32,33]$ and aging [33, 34], suggesting that the brain activation seen with the maintenance of neck flexion may be an acquired factor. However, when such brain activation function is acquired within the period from childhood to adolescence has not yet been reported. The ascending activation system is known to consist of two subsystems: a dorsal pathway from the reticular formation to the thalamus and prefrontal cortex; and a ventral pathway from the reticular formation to the hypothalamus and prefrontal cortex [35, 36]. Myelination in the reticular formation and frontal lobe, contributing to brain activation, is continuously observed after 10 years old [37-40]. Furthermore, developmental improvement of the performance related to frontal lobe function is observed until a later age, compared with that of pro-saccade performance $[41,42]$. Considering these developmental changes, the appearance of the brain activation with the maintenance of neck flexion would be found from a later age, compared with the age when the informationprocessing function in the pro-saccade enhances.

Furthermore, brain activation in young adults with the maintenance of a neck flexion position, related to the shortening of the pro-saccade reaction time, is reportedly maintained for around $30 \mathrm{~s}$ [43]. However, developmental changes in the continuity of brain activation have never been investigated. The continuity of brain activation might be related to sustained attentional function [43]. The function of sustained attention to maintain early response to a visual target has been suggested to enhance from infant to young adulthood [44, 45]. Considering these findings, brain activation with neck flexion would be sustained longer with age.

The present study investigated developmental changes in shortening of pro-saccade reaction time while maintaining the neck flexion position and the continuity of this shortening. The working hypotheses were as follows. First, the shortening of the pro-saccade reaction time with neck flexion would be found from a later age, compared with the age at which the pro-saccade reaction time decreases. Second, this shortening of reaction time during neck flexion would be sustained longer with age.

\section{Methods \\ Subjects}

Subjects comprised 135 children (age range, 3-14 years), and 29 young adults (age range, 19-23 years). Children were divided into 6 groups by every 2-year-old: 13 children in the 3- to 4-year-old group; 31 in the 5- to 6-year-old group; 18 in the 7- to 8-year-old group; 24 in the 9- to 10-year-old group; 21 in the 11- to 12-year-old group and 28 in the 13- to 14-year-old group. No subjects had any history of neurological impairment. In accordance with the Declaration of Helsinki, all parents and young adults provided written informed consent to participate in this study after receiving an explanation of the experimental protocols and how privacy would be protected. All experimental protocols were approved by our institutional ethics committee.

\section{Apparatus and data recording}

The experimental setup is shown in Fig. 1. Subjects sat on a steel-frame chair with the back resting against a vertical wall, and the trunk was secured by a cotton band to prevent antero-posterior movement. The knees were kept flexed at approximately $90^{\circ}$ and the feet were rested on a low table. Neck flexion angle was measured as described by Fujiwara et al. [20]. The flexion angle was defined as the rotational angle of the tragus around the acromion in the sagittal plane, with the starting position $\left(0^{\circ}\right)$ as the neck rest position. We determined $80 \%$ of maximal neck flexion angle (neck flexion position) for each subject using a custom-made angular detector with the center point set at the acromion, while regulating the distance between the acromion and tragus. The set angle in the flexed position was defined as described by Fujiwara et al. and Kunita and Fujiwara [28, 31]. Head inclination angle was determined as the angle between the auriculo-infraorbital line and the gravitational line, and this was maintained at the same angle as the sitting posture to maintain constant sensory inputs from the vestibular organ. An angular detector using the pendulum principle (Level + angle detector; Mitsutomo, Tokyo, Japan) was attached to the temple to confirm this angle. A chin stand was used to support the head and allow maximal relaxation of the neck extensor muscles.

A visual stimulator with light-emitting diodes (LEDs) (SLE-5100; Nihon Kohden, Tokyo, Japan) was used to induce saccadic eye movement. LEDs were located at the central fixation point and at the targets and were illuminated for time periods set by a functional 


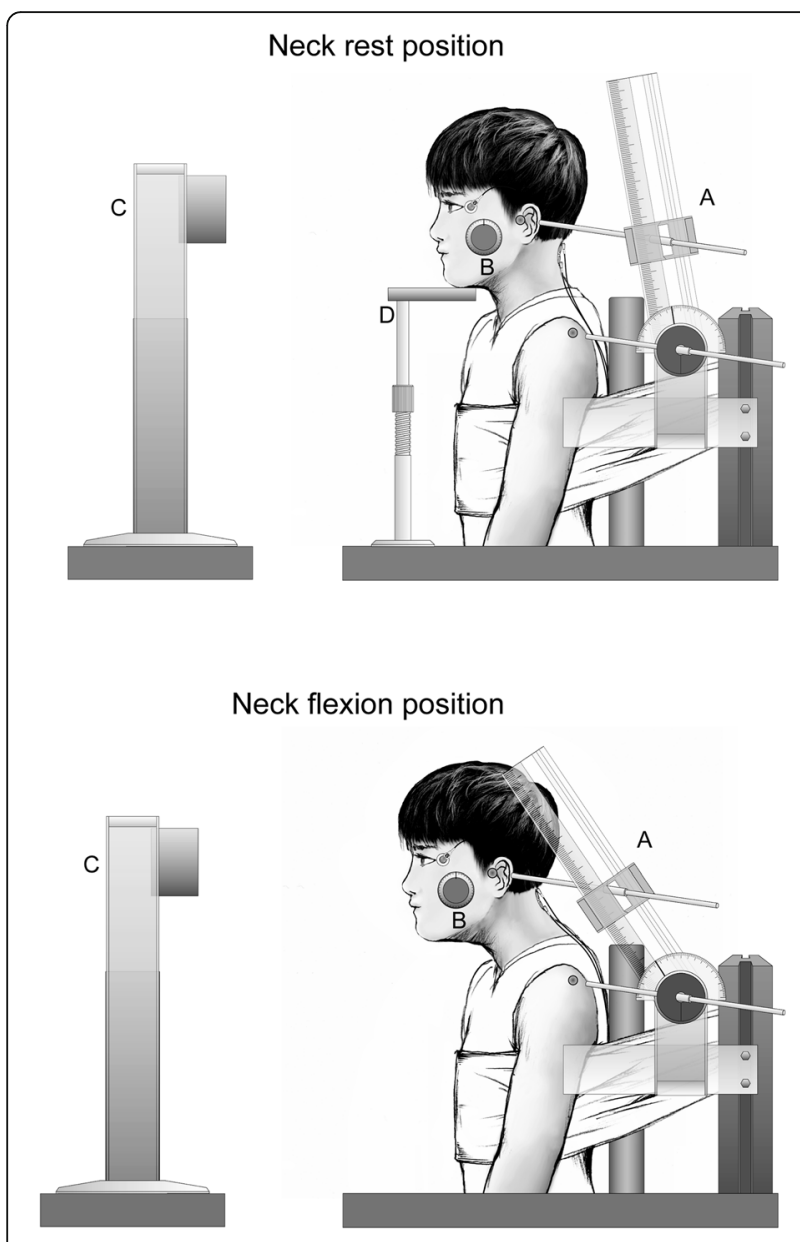

Fig. 1 Experimental setup. A: Neck angular detector. B: Head inclination angular detector using the pendulum principle. C: Visual stimulator. D: Chin stand

generator (WF1966; NF, Kanagawa, Japan). The size of the LED was $0.23^{\circ}$ in height and $0.80^{\circ}$ wide, with illuminance of 0.02 lumen. LEDs were placed at the height of the nose root, and the distance between the LED at the central fixation point and the nose root was set at $50 \mathrm{~cm}$. The central fixation point was illuminated for a random duration of 2-4 s, and one of the lateral targets was subsequently illuminated for $1 \mathrm{~s}$ (Fig. 2). The four lateral targets were located at $5^{\circ}$ and $10^{\circ}$ to the left and right of the central fixation point, and were presented with equal probability at random.

Horizontal eye movements and blinks were measured using electrooculography (EOG). Horizontal EOG was recorded from surface electrodes (P-00-S; Ambu, Ballerup, Denmark) at the outer canthus of each eye, and vertical EOG from electrodes above and below the left eye. A ground electrode was placed at the center of the forehead. Electrode-input impedance was reduced to $<10 \mathrm{k} \Omega$. The signal from the electrodes was amplified (×2000) using a DC amplifier (AN-601G; Nihon Kohden,
Tokyo, Japan). To obtain stable EOG traces, recording began at least $20 \mathrm{~min}$ after the placement of electrodes. Surface electrodes with bipolar derivation were used to monitor and record surface electromyography (EMG) activity of the bilateral upper trapezius muscles. Interelectrode distance was about $3 \mathrm{~cm}$. A ground electrode was placed at the spinous process of the $\mathrm{C} 7$ vertebra. Electrode impedances were reduced to $<5 \mathrm{k} \Omega$. Signals from these electrodes for trapezius were amplified $(\times 2000)$ and band-pass filtered $(5-500 \mathrm{~Hz})$, using an EMG amplifier (MA1000; DIGITEX, Tokyo, Japan). EOG and visual stimulus data were sent to a computer (Dimension E521; Dell, Kawasaki, Japan) via an A/D converter (ADA16-32/2(CB) F; Contec, Osaka, Japan) at $1000 \mathrm{~Hz}$ with 16-bit resolution. EMG data were sent to an oscilloscope (DS-6612; Iwatsu, Tokyo, Japan) to monitor activation.

\section{Procedure}

Pro-saccade reaction task was carried out under neck rest and neck flexion positions. Initially, to familiarize subjects with pro-saccade, 20 -s practice trials in the neck rest position were performed three times. Next, 30-s experimental trials were repeated five times in each postural condition. Prior to beginning each trial, contraction and relaxation of the trapezius muscles were alternated several times and deep breaths was taken to relax the muscle. The experimenter verbally instructed the subject to relax the trapezius muscle, with monitored by EMG. After relaxation of the muscle was confirmed, LED illumination was start. Subjects were instructed to turn their gaze as quickly as possible to the illuminated target. In the neck flexion position, the subject first performed neck flexion within approximately $3 \mathrm{~s}$, maintained this position for $2 \mathrm{~s}$, and then started each trial. Neck angles and head inclination angle during the saccadic task were monitored from the left side by an experimenter. Rest periods of $1 \mathrm{~min}$ and $3 \mathrm{~min}$ were provided between trials and between conditions, respectively. The order of neck position conditions was counterbalanced across subjects to consider potential effects of fatigue and sequential learning.

\section{Data analysis}

Analysis of visual stimuli, pro-saccade, and EMGs of the trapezius were performed using BIMUTAS-II software (Kissei Comtec, Matsumoto, Japan). Pro-saccade reaction time was defined as the latency between onset of target movement and beginning of eye movement (Fig. 3). Onset of eye movement was determined by visual inspection of EOG displacement that was easily discernible from baseline. The pro-saccade reaction time for each condition was averaged for every 10 -s period. 

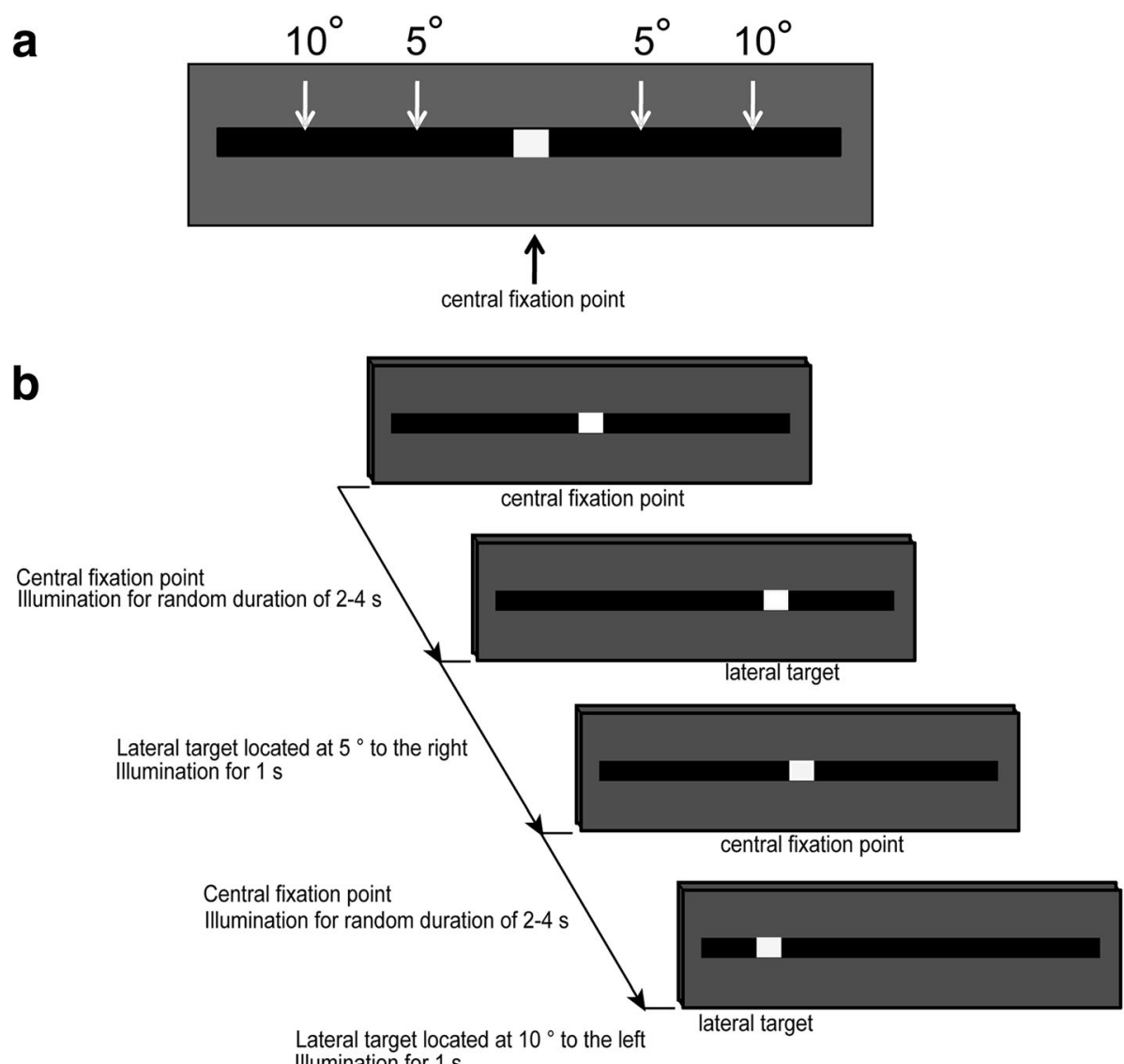

Fig. 2 a Four lateral targets located at $5^{\circ}$ and $10^{\circ}$ to the left and right of the central fixation point. $\mathbf{b}$ Presentation protocol in the pro-saccade task

To analyze the activity level of upper trapezius muscles, all EMGs were 40-Hz high-pass filtered using the seventhorder Butterworth method to exclude electrocardiographic and movement artifacts, then full-wave rectified. In each neck position, mean EMG amplitude of each side of the upper trapezius muscle for every 10-s period was calculated. Thereafter, relative ratio of the EMG amplitude of the neck flexion condition against the neck rest condition was calculated using the following equation for each trapezius muscle in each 10 -s period:

Relative ratio $=($ EMGnx - EMGmin $) /($ EMGrx - EMGmin $)$

where EMGnx is EMG mean amplitude at the neck flexion position in each of the 15 time periods ( 5 trials $\times$ 3 time periods), EMGmin is the smallest value in mean EMG amplitude at the neck rest position, and EMGrx is the mean EMG amplitude at the neck rest position in each of the 15 time periods. After that, relative ratios from right and left sides in 5 trials were averaged separately for each 10 -s period.

\section{Statistical analysis}

Data were tested for normality using the Shapiro-Wilks test and for equality of variance using Leven's test. Two-way analysis of variance (ANOVA) was used to assess the effect of age (3- to 14-year-old children and young adults) and time period (0-10 s, 10-20 s and 20-30 s) on relative ratio of EMG amplitude. Twoway repeated-measures ANOVA was used to assess the effect of time period and neck position (neck rest and flexion positions) on saccade reaction time in each age group. When significant main effects of time period and neck position were recognized, the post hoc Tukey's honestly significant difference (HSD) test was used to investigate differences within the time period and the Bonferroni-adjusted paired t-test was used to investigate differences within neck position, respectively. One-way ANOVA was used to compare reaction times for the $0-10 \mathrm{~s}$ period in the neck rest position across age groups. Post hoc comparisons were performed using Tukey's HSD test to further examine differences suggested by ANOVA. The alpha level was set at $p<0.05$. All statistical 


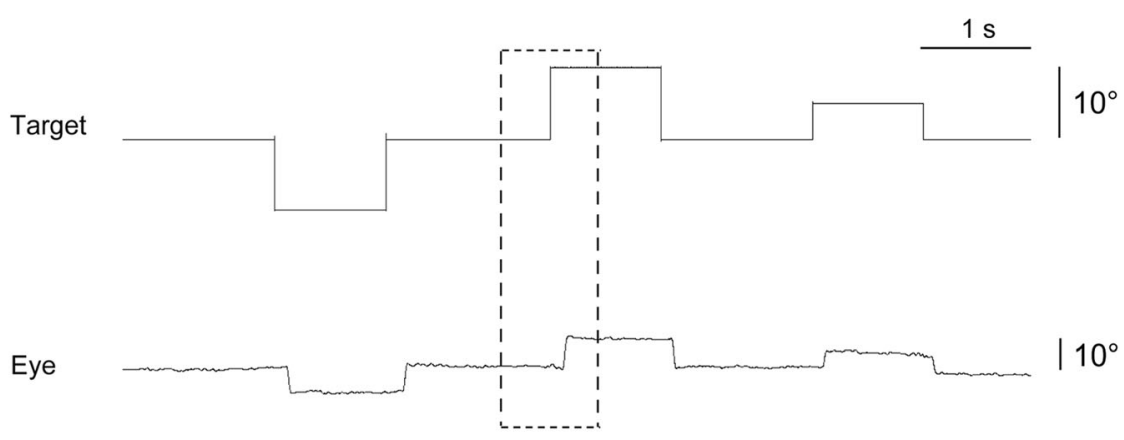

Neck rest position

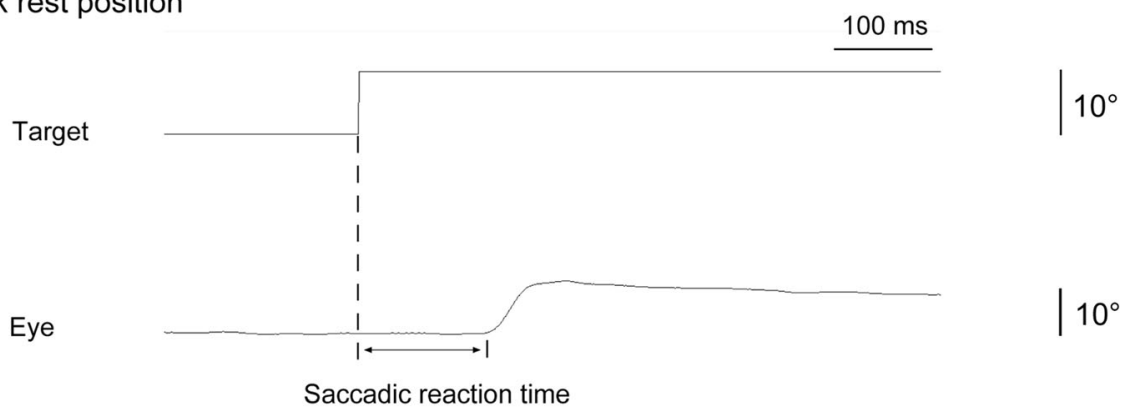

Neck flexion position

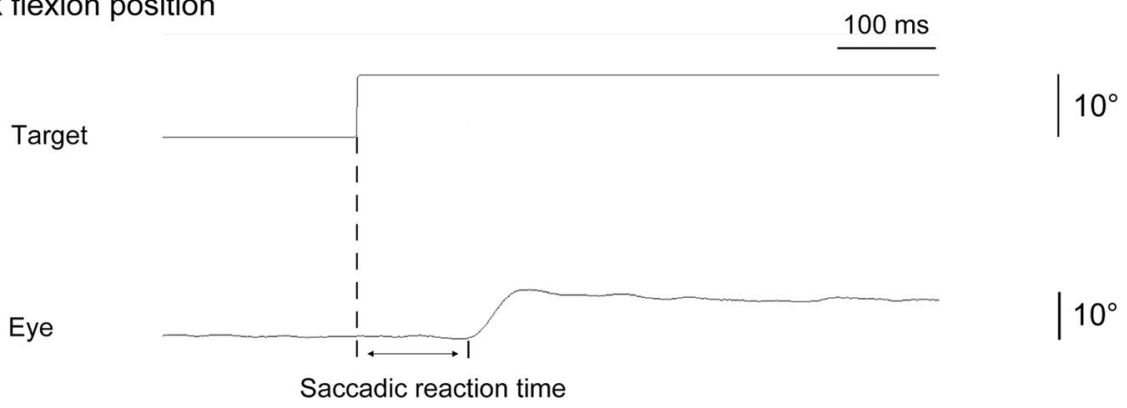

Fig. 3 Analysis of saccadic reaction time in two sample records (neck rest position and neck flexion position)

analyses were performed using IBM SPSS Statistics version 21 (IBM Japan, Tokyo, Japan).

\section{Results}

Means and standard deviations of relative ratio of EMG mean amplitude in the trapezius are shown in Fig. 4. The relative ratio was within the range from 5.20 to 6.57, and no significant main effect of age or time period, or interaction between these factors was found in the relative ratio.

Means and standard deviations of pro-saccade reaction time in each 10-s period are shown in Fig. 5. For all groups from 3 to 10 years old, only a significant main effect of time period was found in the reaction time (3- to 4-year-old group: $F_{2,24}=5.33, p<0.05 ; 5$ - to 6-yearold group: $F_{2,60}=23.73, p<0.001 ; 7$ - to 8 -year-old group: $F_{2,34}=3.90, p<0.05$; 9 - to 10 -year-old group: $F_{1.60,36.75}=$ $3.66, p<0.05)$. For the 3 - to 4 -year-old and 5- to 6-yearold groups, reaction time was significantly longer in the order of $20-30 \mathrm{~s}, 10-20 \mathrm{~s}$ and $0-10 \mathrm{~s}$ periods $(p s<0.05)$. For the 7 - to 8 -year-old group, reaction time was significantly longer in the $10-20 \mathrm{~s}$ and $20-30 \mathrm{~s}$ periods than in the $0-10 \mathrm{~s}$ period $(p s<0.05)$. For the 9 - to 10 -year-old group, reaction time was significantly longer in the 20$30 \mathrm{~s}$ period than in the $0-10 \mathrm{~s}$ period $(p<0.05)$. For the 11 - to 12-year-old group, significant main effects of time period $\left(F_{1.56,31.16}=7.08, \quad p<0.01\right)$ and neck position $\left(F_{1,20}=12.12, p<0.01\right)$ were found, but no significant 

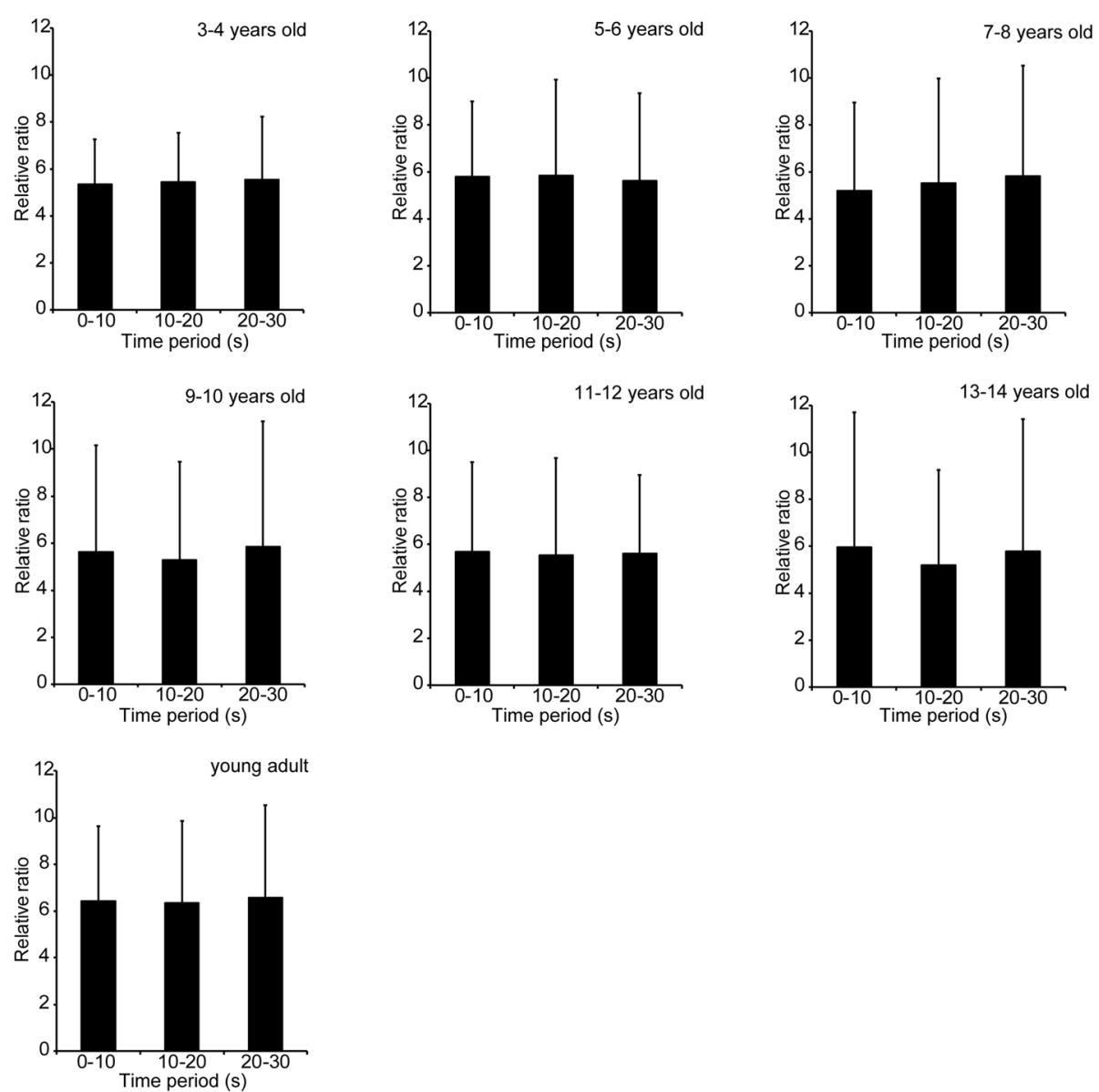

Fig. 4 Mean and standard deviation of relative ratio of EMG amplitude in every 10-s time band

interaction was apparent. Although no significant differences in reaction time were found in the neck rest position, reaction time at the neck flexion position was significantly longer in the $20-30 \mathrm{~s}$ period than in the other two periods $(p s<0.05)$. In the $0-10 \mathrm{~s}$ and $10-20 \mathrm{~s}$ periods, reaction time was significantly shorter at the neck flexion position than in the neck rest position ( $p s<0.05$ ). For the 13- to 14-year-old and young adult groups, the only significant main effect of neck position was found in the reaction time (13- to 14year-old group: $F_{1,27}=43.97, p<0.001$; young adults: $\left.F_{1,28}=57.44, p<0.001\right)$. In every time period, reaction time was significantly shorter in the neck flexion position than in the neck rest position $(p s<0.01)$.

To investigate age-related changes in reaction time at the neck rest position regardless of time-dependent prolongation, we compared reaction times for the $0-10 \mathrm{~s}$ period among all age groups. A significant effect of age was found in reaction time at the neck rest position for the $0-10 \mathrm{~s}$ period $\left(F_{6,60.5}=5.50, p<0.01\right)$. Reaction time was significantly longer in the 3 - to 4 -year old group than in the 5- to 6-year-old group and above
( $p s<0.01)$. No significant differences in reaction time were observed among the 5- to 6-year-old group and above.

\section{Discussion}

Significant time-dependent prolongations in pro-saccade reaction time at the neck rest position were found for all groups from 3 to 10 years old. Age-related changes at the neck rest position were then examined, using the reaction time for the $0-10 \mathrm{~s}$ period. This reaction time for the $0-10 \mathrm{~s}$ period was significantly longer in the 3to 4-year-old group than in the 5- to 6-year-old group and above. No significant age difference in reaction time for the $0-10 \mathrm{~s}$ period was observed among the 5- to 6year-old group and above. These results indicate that age-related enhancement of information-processing function in the pro-saccade was observed until at least 5-6 years old, by which the function reached similar levels to young adults, in line with the findings of previous studies [8, 11, 46, 47].

Significant shortening of pro-saccade reaction time with neck flexion position was not found until the 9- to 10-year-old group, but was evident in the 11- to 12-year- 


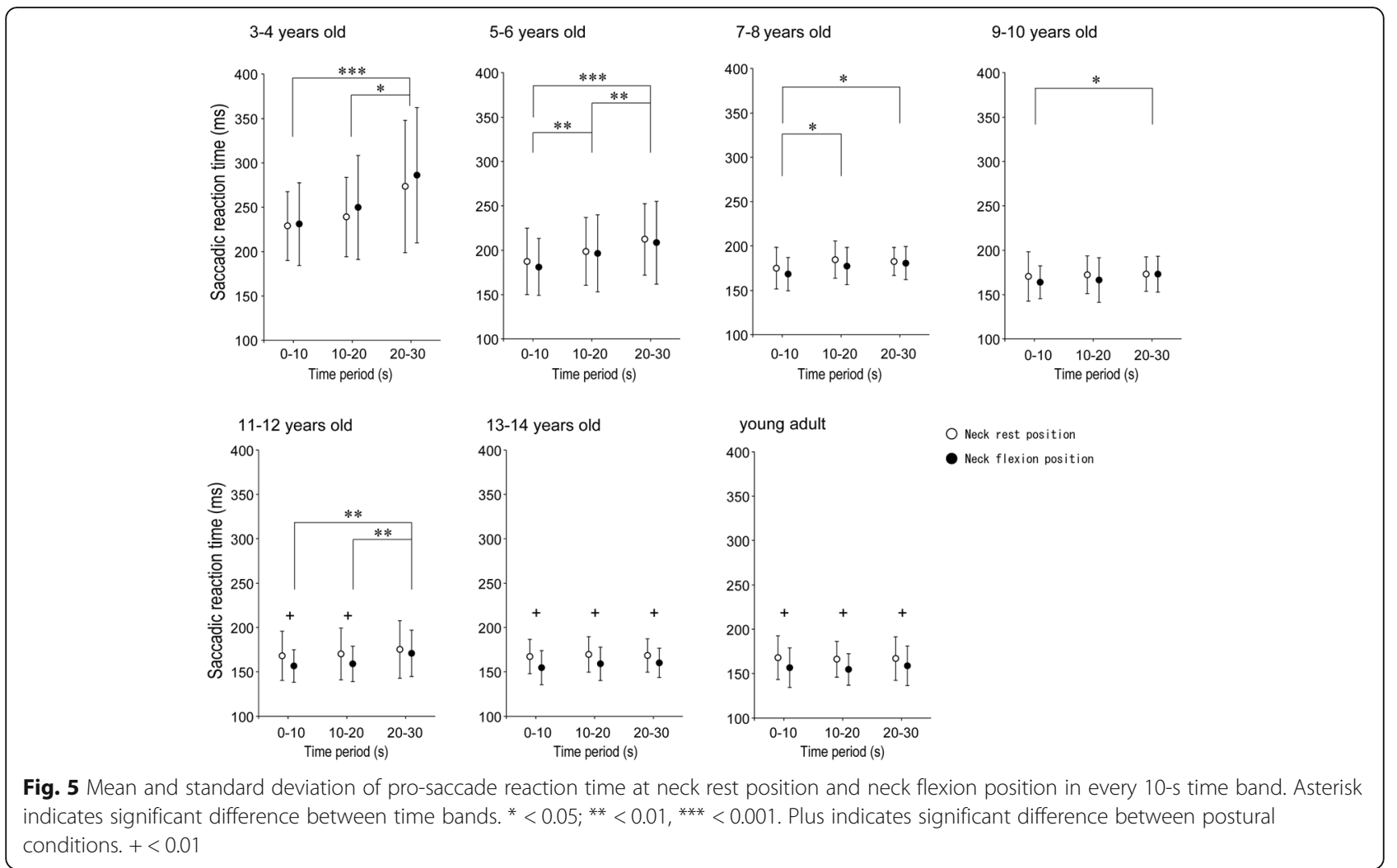

old group and above. These findings suggest that brain activation with maintenance of the neck flexion position would be acquired after approximately 11 years old. No significant age effect was found in relative ratio of EMG mean amplitude in the trapezius. This indicated that muscle activity levels of the neck extensors were unrelated to developmental changes in the shortening of prosaccade reaction time while maintaining neck flexion. Myelination of the brainstem reticular formation and frontal lobe, contributing to brain activation, has been reported to be observed after 10 years old [37-40]. Furthermore, age-related improvements in performances related to frontal lobe function have been suggested to start later than those of pro-saccade performance $[41,42]$. Relating to such developmental differences in brain function, the results in the present study suggest that the appearance of brain activation while maintaining a neck flexion position, which causes shortening of the pro-saccade reaction time, was found from a later age, compared with the age when the information-processing function in the prosaccade enhanced.

The significant shortening of the pro-saccade reaction time with neck flexion was sustained until 20-s period in the 11- to 12-year-old-group and during the entire $30 \mathrm{~s}$ in the 13- to 14-year-old and young adult groups. This result suggests that age-related marked enhancement of sustained brain activation with the neck flexion was observed even later, at approximately 13 years and above, compared with the age at which brain activation with neck flexion appeared. The result that no significant effect of time period was found in the relative ratio of EMG amplitude suggests that time-dependent changes in muscle activity of the neck extensors were unrelated to development of sustained brain activation. Such sustained brain activation might be related to attentional function [43]. Sustained attention to maintain early response to appearing visual targets increases from infancy to early adulthood $[44,45]$. In particular, sustained attention has been reported to be markedly enhanced after 13-14 years [45]. Considering these findings, marked enhancement of sustained attention after 13 years might be related to sustained brain activation with the maintenance of neck flexion.

\section{Conclusions}

The results in the present study suggested that the brain activation with the maintenance of neck flexion, related to shortening of the pro-saccade reaction time, was found from a later age at approximately 11 years and above, compared with the age at which informationprocessing function in the pro-saccade enhanced. In addition, brain activation with neck flexion was suggested to be sustained longer with age. 


\section{Abbreviations}

ANOVA: Analysis of variance; EMG: Electromyography:

EOG: Electrooculography; HSD: Honestly significant difference; LED: Lightemitting diode

\section{Acknowledgements}

We are grateful to the participants of the present study.

\section{Funding}

This work was supported by Grant-in-Aid for Scientific Research from the Japan Society for the Promotion of Science (No.22570227).

\section{Availability of data and materials}

The datasets used and/or analyzed during the current study available from the corresponding author on reasonable request.

\section{Authors' contributions}

The contribution of each author is as follows. KK and KF presented all the idea of this study, planed the method, directed the experiments and interpreted the results. NK, CY, and TK contributed to the experiments, data analyses, and manuscript preparation. All authors read and approved the final manuscript.

\section{Ethics approval and consent to participate}

In accordance with the Declaration of Helsinki, all subjects provided informed consent after receiving an explanation of the experimental protocol, which was approved by the ethics committee at Sapporo International University.

\section{Consent for publication \\ Not applicable.}

\section{Competing interests}

The authors declare that they have no competing interests.

\section{Publisher's Note}

Springer Nature remains neutral with regard to jurisdictional claims in published maps and institutional affiliations.

\section{Author details}

${ }^{1}$ Department of Sports Instruction, Faculty of Sports and Human, Sapporo International University, 4-1-4-1 Kiyota, Kiyota-ku, Sapporo 004-8602, Japan. ${ }^{2}$ Department of Sports and Health, Kanazawa Gakuin University, 10 Sue-machi, Kanazawa 920-1392, Japan. ${ }^{3}$ Department of Rehabilitation, Japan Health Care College, 6-17-3 Megumino-nishi, Eniwa 061-1373, Japan.

Received: 18 August 2017 Accepted: 26 December 2017

\section{Published online: 10 January 2018}

\section{References}

1. Carter N, Zee DS. The anatomical localization of saccades using functiona imaging studies and transcranial magnetic stimulation. Curr Opin Neurol. 1997;10:10-7.

2. Hikosaka O, Takikawa Y, Kawagoe R. Role of the basal ganglia in the control of purposive saccadic eye movements. Physiol Rev. 2000;80:953-78.

3. Leigh RJ, Kennard C. Using saccades as a research tool in the clinical neurosciences. Brain. 2004;127:460-77.

4. Pierrot-Deseilligny C, Rivaud S, Gaymard B, Müri R, Vermersch A-I. Cortical control of saccades. Ann Neurol. 1995;37:557-67.

5. Schall JD, Thompson KG. Neural selection and control of visually guided eye movements. Annu Rev Neurosci. 1999;22:241-59.

6. Fischer B, Biscaldi M, Gezeck S. On the development of voluntary and reflexive components in human saccade generation. Brain Res. 1997; 754:285-97.

7. Fukushima J, Hatta T, Fukushima K. Development of voluntary control of saccadic eye movements. I. Age-related changes in normal children. Brain Dev. 2000;22:173-80

8. Irving EL, Steinbach MJ, Lillakas L, Babu R, Hutchings N. Horizontal saccade dynamics across the human life span. Invest Ophthalmol Vis Sci. 2006;47:2478-84.
9. Karatekin C. Eye tracking studies of normative and atypical development. In: Nelson CA, Luciana M editors, Handbook of developmental cognitive neuroscience 2nd ed. Cambridge: MIT Press: 2008. p 263-299.

10. Klein C. Developmental functions for saccadic eye movement parameters derived from pro- and antisaccade tasks. Exp Brain Res. 2001;139:1-17.

11. Klein C, Foerster F. Development of prosaccade and antisaccade task performance in participants aged 6 to 26 years. Psychophysiology. 2001; 38:179-89.

12. Klein C, Rauh R, Biscaldi M. Patterns of change in ocular motor development. Exp Brain Res. 2011;210:33-44.

13. Munoz DP, Broughton JR, Goldring JE, Armstrong IT. Age-related performance of human subjects on saccadic eye movement tasks. Exp Brain Res. 1998:121:391-400.

14. Luna B, Velanova K, Geier CF. Development of eye-movement control. Brain Cogn. 2008;68:293-308.

15. Coull JT. Neural correlates of attention and arousal: insights from electrophysiology, functional neuroimaging and psychopharmacology. Prog Neurobiol. 1998;55:343-61.

16. Coull JT, Jones MEP, Egan TD, Frith CD, Maze M. Attentional effects of noradrenaline vary with arousal level: selective activation of thalamic pulvinar in humans. Neuroimage. 2004;22:315-22.

17. Fimm B, Willmes K, Spijkers W. The effect of low arousal on visuo spatial attention. Neuropsychologia. 2006:44:1261-8.

18. Portas CM, Rees G, Howseman AM, Josephs O, Turner R, Frith CD. A specific role for the thalamus in mediating the interaction of attention and arousal in humans. J Neurosci. 1998;18:8979-89.

19. Howorth B. Dynamic Posture. J Am Med Assoc (Chicago). 1946;131:1398-404

20. Fujiwara K, Kunita K, Toyama $H$. Changes in saccadic reaction time while maintaining neck flexion in men and women. Eur J Appl Physiol. 2000:81:317-24.

21. Fujiwara K, Kunita K, Watanabe $H$. Sports exercise effect on shortening of saccadic reaction time associated with neck extensor muscle activity. Int J Sports Med. 2006;27:792-7.

22. Fujiwara K, Kunita K, Toyama H. Latency of saccadic eye movement during contraction of bilateral and unilateral shoulder girdle elevators. Percept Mot Skills. 2003:96:173-84.

23. Fujiwara K, Kunita K, Toyama H, Miyaguchi A. Saccadic reaction time during isometric voluntary contraction of the shoulder girdle elevators and vibration stimulation to the trapezius. Eur J Appl Physiol. 2001;85:527-32.

24. Fujiwara K, Kunita K, Furune N. Effect of vibration stimlation to neck extensor muscles on reaction time in various saccadic eye movements. Int J Neurosci. 2009;119:1925-40.

25. Berthoz A. The role of inhibition in the hierarchical gating of executed and imagined movements. Brain Res Cogn Brain Res. 1996;3:101-13.

26. Shimura K, Kasai T. Effects of proprioceptive neuromuscular facilitation on the initiation of voluntary movement and motor evoked potentials in upper limb muscles. Hum Mov Sci. 2002;21:101-13.

27. Vallar G, Rusconi ML, Barozzi S, Bernardini B, Ovadia D, Papagno C, Cesarani A. Improvement of left visuo-spatial hemineglect by left-sided transcutaneous electrical stimulation. Neuropsychologia. 1995;33:73-82.

28. Fujiwara K, Tomita H, Maeda K, Kunita K. Effects of neck flexion on contingent negative variation and anticipatory postural control during arm movement while standing. J Electromyogr Kinesiol. 2007;19:113-21.

29. Fujiwara K, Kunita K, Kiyota N, Mammadova A, Irei M. The effects of neck flexion on cerebral potentials evoked by visual, auditory and somatosensory stimuli and focal brain blood flow in related sensory cortices. J Physiol Anthropol. 2012;31:31

30. Fujiwara K, Yaguchi C, Kunita K. Effects of neck flexion on discriminative and cognitive processing in anticipatory postural control during bilateral arm movement. Neurosci Lett. 2012;518:144-8.

31. Kunita K, Fujiwara K. Effect of maintaining neck flexion on anti-saccade reaction time: an investigation using transcranial magnetic stimulation to the frontal oculomotor field. J Physiol Anthropol. 2013;32:21.

32. Kunita K, Fujiwara K. Neck-shortening effect on prosaccade reaction time formed through saccadic training accompanied by maintenance of neck flexion. Eur J Appl Physiol. 2009;107:597-602.

33. Kiyota N, Fujiwara K. Effects of anti-saccade training with neck flexion on eye movement performance, presaccadic potentials and prefrontal hemodynamics in the elderly. Eur J Appl Physiol. 2010;110:1143-54.

34. Kunita K, Fujiwara K. Changes in saccadic reaction time while maintaining neck flexion in the elderly. J Physiol Anthropol. 2006;25:257-61. 
35. Jones BE. Activity, modulation and role of basal forebrain cholinergic neurons innervating the cerebral cortex. Prog Brain Res. 2004;145:157-69.

36. Valatx JL. Disorders of consciousness: anatomical and physiological mechanisms. Adv Tech Stand Neurosurg. 2004:29:3-22.

37. Kharitonova M, Martin RE, Gabrieli JD, Sheridan MA. Cortical gray-matter thinning is associated with age-related improvements on executive function tasks. Dev Cogn Neurosci. 2013;6:61-71.

38. Toga AW, Thompson PM, Sowell ER. Mapping brain maturation. Trends Neurosci. 2006;29:148-59.

39. Yakovlev PI, Lecours AR. The myelogenetic cycles of regional maturation of the brain. In: Minkowski A, editor. Regional development of the brain in early life. Oxford: Blackwell; 1967. p. 3-70.

40. Zhong J, Rifkin-Graboni A, Ta AT, Yap KL, Chuang K-H, Meaney MJ, Qiu A. Functional networks in parallel with cortical development associate with executive functions in children. Cereb Cortex. 2014:24:1937-47.

41. Crone EA, Wendelken C, Donohue S, van Leijenhorst L, Bunge SA. Neurocognitive development of the ability to manipulate information in working memory. Proc Natl Acad Sci U S A. 2006;103:9315-20.

42. Velanova $\mathrm{K}$, Wheeler ME, Luna B. Maturational changes in anterior cingulate and frontoparietal recruitment support the development of error processing and inhibitory control. Cereb Cortex. 2008;18:2505-22.

43. Mammadova A, Fujiwara K. Saccadic shortening and cerebral hemodynamics during neck flexion and vibrational stimulation on neck extensor muscles. Health Behav Sci. 2013;11:73-80.

44. Hrabok M, Kerns KA, Müller U. The vigilance, orienting, and executive attention networks in 4-year-old children. Child Neuropsychol. 2007;13:408-21.

45. Lin CCH, Hsiao CK, Chen WJ. Development of sustained attention assessed using the continuous performance test among children 6-15 years of age. J Abnorm Child Psychol. 1999;27:403-12.

46. Cohen ME, Ross LE. Saccade latency in children and adults: effects of warning interval and target eccentricity. J Exp Child Psychol. 1977;23:539-49.

47. Cohen ME, Ross LE. Latency and accuracy characteristics of saccades and corrective saccades in children and adults. J Exp Child Psychol. 1978;26:517-27.

\section{Submit your next manuscript to BioMed Central and we will help you at every step:}

- We accept pre-submission inquiries

- Our selector tool helps you to find the most relevant journal

- We provide round the clock customer support

- Convenient online submission

- Thorough peer review

- Inclusion in PubMed and all major indexing services

- Maximum visibility for your research

Submit your manuscript at www.biomedcentral.com/submit

) Biomed Central 